\title{
Awareness of everyday executive difficulties precede overt executive dysfunction in schizotypal subjects
}

\author{
Keith R. Laws ${ }^{\text {a,* }}$, Devina D. Patel ${ }^{\text {a }}$, Philip J. Tyson ${ }^{\text {b }}$ \\ a School of Psychology, University of Hertfordshire, College Lane Hatfield, AL10 9AB, UK \\ ${ }^{\mathrm{b}}$ School of Biological and Behavioural Sciences, University of Gloucestershire, UK
}

Received 7 November 2006; received in revised form 19 March 2007; accepted 3 June 2007

\begin{abstract}
Much evidence indicates that schizophrenic patients exhibit deficits on tests of executive functioning. It is therefore hypothesized that individuals with high schizotypal personality traits that may have a predisposition to schizophrenia, are also likely to exhibit impairments in neuropsychological tests of executive function. The sample consisted of $61_{\Lambda}$ healthy controls that were divided into high and low scorers on the Schizotypal Personality Questionnaire (SPQ-B: Raine et al., 1995). Participants completed a battery of executive tasks (category and letter fluency, the Hayling test, Zoo map); however, a MANOVA revealed no significant differences between high and low SPQ scorers. Nevertheless, high SPQ scorers scored significantly higher on the dysexeeutive (DEX) self-rating scale of everyday executive problems; and these self-ratings correlated significantly with the disorganisation and cognitive-perceptual features of the SPQ-B, but not with the interpersonal features. This suggests that perceived executive dysfunction is pre-morbidly present and may become evident in test performance only with the onset of schizophrenia itself. (C) 2007 Published by Elsevier Ireland Ltd.
\end{abstract}

Keywords: Schizotypy; Schizophrenia; Frontal lobes; Dysexecutive syndrome; Awareness

\section{Introduction}

Executive function is a broad term which encompasses the cognitive processes involved in the control and regulation of goal selection, higher order inferences and problem solving. Executive systems allow us to inhibit immediate responses, flexibly switch between problem-solving strategies, plan and execute strategies, monitor complex behavioural sequences and to re-direct and sustain attention flexibly. Much evidence shows that patients with schizophrenia are impaired on a wide

\footnotetext{
* Corresponding author.

E-mail address: k.laws@herts.ac.uk (K.R. Laws).
}

range of tasks believed to tap such executive functions 32 (for a review, see Laws, 1999). A meta-analytic review 33 (Heinrichs and Zakzanis, 1998) comparing the perfor- 34 mance of patients with schizophrenia and controls has 35 revealed large effect sizes on the most widely-used tests 36 of executive function: fluency $(d=1.39)$, Stroop Colour 37 Word Test $(d=1.22)$, Trail Making Test B (TMT-B: 38 $d=1.07$ ), and the Wisconsin Card Sort Test (WCST: 39 $d=.95)^{1}$. The nomenclature of Cohen (1988) suggests 40 the following classification of effect sizes (small $d=41$

\footnotetext{
${ }^{4}$ The nomenclature of Cohen (1988) suggests the following elassification of effect sizes (small $d-0.20$; medium $d-0.50$; and large $d=0.80$ ).
} 
0.20 ; medium $d=0.50$; and large $d=0.80)$.. It is argued that executive functioning is mediated primarily by the prefrontal lobes; and a meta-analysis of functional brain imaging studies in schizophrenic patients points to reduced blood flow and metabolism in their frontal cortex when performing executive tests (Hill et al., 2004 for a meta-analysis). Furthermore, meta-analysis of executive functioning in unaffected relatives of schizophrenics reveals moderate effect sizes (TMT-B $d=.51$; fluency $d=.35$; Stroop $d=.28$; WCST $d=.29$ ) and has been proposed as putative endophenotypic markers for schizophrenia (Sitskoorn et al., 2004; Szöke et al., 2005).

Executive functioning has also been examined in healthy volunteers (typically undergraduates) who are psychometrically classified as psychosis-prone according to their scores on measures of schizotypy. Raine (2006) has reviewed over 250 studies of schizotypal subjects revealing difficulties across a variety of domains including executive function as well as sustained attention, working memory, verbal and spatial learning and memory, latent inhibition, negative priming, hemisphere asymmetry, and motor ability. Compared to healthy controls, studies report that schizotypal subjects show an increase in perseverative errors (Spaulding et al., 1989; Raine et al., 1992a; Lenzenweger and Korfine, 1994; Poreh et al., 1995; Suhr, 1997; Daneluzzo et al., 1998; Gooding et al., 1999; Tallent and Gooding, 1999), fewer completed categories and more failures in maintaining set (Lyons et al., 1991; Gooding et al., 1999). In those studies reporting deficits in schizotypal subjects, the effect size for percentage perseverative errors has ranged from moderate $(d=.55 \mathrm{Suhr}$ et al., 1995) to large $(d=.99$ Poreh et al., $1995 ; d=.97$ Daneluzzo et al., 1998) and so, falls midway between the effect sizes generally reported for schizophrenic patients and for their relatives. Poreh et al. (1995) also found that high schizotypals performed significantly worse on the Trail Making Test (TMT) part B, but not for Design Fluency. Suhr (1997) also reported significantly worse performance by high schizotypals on the Stroop. Similarly, individuals clinically diagnosed with schizotypal personality disorder have also shown a greater degree of executive impairment on the WCST than healthy controls (Trestman et al., 1995; Vogelmaier et al., 1997; Diforio et al., 2000). Nevertheless, not all studies have found executive dysfunction in schizotypal subjects on the WCST (Condray and Steinhauer, 1992; Raine et al., 1992b; Lin et al., 2000; Jahshan and Sergi, 2007) or indeed, on other tests of executive function, including the Stroop (Spitznagel and Suhr, 2002), TMT (Suhr, 1997; Mitropoulou et al., 2002; Spitznagel and Suhr, 2002), verbal and semantic fluency (Trestman et al., 1995; Diforio et al., 2000; Kiang and Kutos, 2006) 94 and the Tower of Hanoi/London task (Suhr, 1997; Di- 95 forio et al., 2000).

Despite the often-significant cognitive problems 97 associated with having frontal lesions, it is notable that 98 patients with frontal lobe lesions tend to underestimate 99 their everyday executive difficulties (as measured by the 100 dysexecutive (DEX) questionnaire: Wilson et al., 1996). 101 In a similar vein, patients with schizophrenia often show 102 a lack of insight and furthermore, that this shows a small 103 but significant relationship with executive dysfunction 104 (for a meta-analysis, see Aleman et al., 2006). A pre- 105 vious study using the DEX along with yarious executive 106 measures in patients with schizophrenia, revealed no 107 association between their impaired executive test per- 108 formance and relatively good DEX self-ratings (Evans 109 et al., 1997). Poor awareness of deficit on the DEX has 110 also been found to correlate with poor executive func- 111 tioning in patients with brain injury (Wilson et al., 112 1996). The DEX self-perception measure has not been 113 used in schizotypal subjects; however, given the lack of 114 insight in patients with schizophrenia, we might expect 115 high schizotypal subjects to also show lowered aware- 116 ness of any executive difficulties (as might be revealed 117 on standardised tests).

118

As little doubt remains that executive functioning is 119 one of the most impaired cognitive abilities in schi- 120 zophrenia, it is important to determine if similar, but 121 milder forms of executive dysfunction appear in schizo- 122 typal subjects (or unaffected relatives of schizophrenics). 123 This psychometric high-risk approach is advantageous 124 because it avoids the potential confounds of medication, 125 lengthy hospitalization, and florid symptoms associated 126 with studies of schizophrenic patients. The main aim of 127 the current study is to test whether non-psychotic indi- 128 viduals who score high and on the Schizotypal Per- 129 sonality Questionnaire (SPQ-B: Raine and Benishay, 130 1995), show significantly worse performance on exec- 131 utive functioning tests when compared to individuals 132 with a low SPQ-B scores; and to examine for differences 133 in perceived everyday executive difficulties.

\section{Method}

\subsection{Participants}

A convenience sample of 65 undergraduate students 137 (10 males and 55 females) aged between 18 and 48 years 138 of age $(M=22.0 ;$ S.D. $=6.4$ years $)$ participated in the 139 study. Participants were excluded if English was their 140 second language, or they reported any history of head 141 injury that may have resulted in unconsciousness or 142 
psychiatric illness history. The local ethical committee at the University of Hertfordshire approved the study.

\subsection{Materials and procedure}

All participants completed the following test battery: the Schizotypal Personality Questionnaire, Category and Letter fluency, the Zoo map test, the Hayling test and finally, the DEX questionnaire. These measures were chosen to cover a range of executive functions (e.g. Hayling - initiation speed and response suppression; Fluency — strategic retrieval processes and monitoring; Zoo map - planning) and to utilize time-based dependent variables to more sensitively tap the presence of executive problems.

\subsubsection{Schizotypal Personality Questionnaire (SPQ-B. Raine and Benishay, 1995)}

The SPQ-B (Raine and Benishay, 1995) is a 22 item dichotomous (yes-no) questionnaire derived from the larger SPQ questionnaire (Raine, 1991). The statements in the SPQ-B are based on the DSM-IIIR diagnostic criteria for schizotypal personality disorder. The questionnaire taps three main factors of the schizotypal personality: cognitive-perceptual ( 8 items) e.g. When shopping do you get the feeling that other people are taking notice of you?; interpersonal (8 items) e.g. Do you feel that you are unable to get "close" to people?; and disorganised (6 items) symptoms e.g. I sometimes use words in unusual ways'.

The three factors and total score from the SPQ-B have internal reliabilities ranging from .72 to .80 , correlations with the full 74-item SPQ range from .89 to .94 , and test-retest reliabilities across a two month interval between .86 and .95 (Axelrod et al., 2001; Raine and Benishay, 1995). Correlation between the SPQ-B and clinical interview measures of schizotypal personality disorder is good (ranging from .63 to .73). The original mean SPQ-B reported by Raine and Benishay (1995) was 9.6[S.D. = 5.3]; however, more recent studies suggest a smaller mean, e.g. Compton et al. (2007) reported $M=5.2$ [S.D. $=4.1]$ and Mata et al. (2005) reported $M=7.3[$ S.D. $=4.2]$. Mata et al. documented their top $10 \%$ as scoring $12+$ and the lowest $10 \%$ scoring $<3$.

\subsubsection{Category and letter fluency test (Goodglass and} Kaplan, 1972; Benton and Hamsher, 1976)

These fluency tests measured the number of words generated in one minute. Four fluency tests were administered: two category tests 'animals' and 'fruits' and two letter tests ' $\mathrm{F}$ ' and ' $\mathrm{S}$ '.
2.2.3. Zoo map subtest (Behavioural Assessment of the 191 Dysexecutive Syndrome: Wilson, Alderman, Burgess, 192 Emslie, and Evans, 1996)

The Zoo map was used to assess the ability to in- 194 dependently formulate and implement plans. In this test, 195 participants are given a map of a zoo, a set of instruc- 196 tions describing places they need to visit in the zoo (e.g., 197 elephant house, lion's cage) and rules they must not 198 contravene (e.g. starting at the entrance and finishing at 199 the picnic area, without using unshaded paths more than 200 once and by only taking one camel ride). The experi- 201 menter recorded the amount of time spent planning and 202 drawing (i.e. executing) a route.

203

2.2.4. Hayling sentence completion task (Burgess and 204 Shallice, 1997)

The Hayling sentence completion test was adminis- 206 tered to participants to measure response inhibition. The 207 test involves hearing a sentence which the participant 208 must complete with one word. In one condition (Auto- 209 matic sentence), the sentence is completed by a mean- 210 ingful word e.g. "The old house will be torn ......." - a 211 correct response would be "down". In the second con- 212 dition, the participant provides a word that is completely 213 unconnected to the sentence (Inhibition sentence), e.g. 214 "None of the books made any ......." a possible response 215 might be 'button'. The word produced and the response 216 time was recorded by the researcher on a response sheet 217 for both sections. The total response time for all 15 items 218 in each condition (automatic and inhibition) was the 219 performance measure.

\subsubsection{Dysexecutive questionnaire (Behavioural Assess- 221} ment of the Dysexecutive Syndrome: Wilson, Alderman, 222 Burgess, Emslie, Evans, 1996)

The DEX questionnaire obtained from the BADS 224 battery of tests consisted of 20 statements that describe 225 behaviour associated with the dysexecutive syndrome. 226 An example of a question from the questionnaire is 'I 227 have problems understanding what other people mean 228 unless they keep things simple and straight forward'. 229 The questionnaire uses a likert self-rating scale ranging 230 from 'never' to 'often' (0-4). Two recent studies (Chan 231 et al., 2001 and Wilson et al., 1996) revealed mean DEX 232 scores of 22.12 (S.D. $=8.86)$ and 20.99 㣏.D. $=9.63 \hbar$ in 233 samples of 93 and 216 healthy subjects respectively. 234

The 20 items assess problems associated with the 235 following four factors derived from 293 healthy subjects 236 (Mooney et al., 2006): inhibition, intention, social 237 regulation and abstract problem solving. Items in the 238 first factor included those questions relating to the in- 239 ability to inhibit behavioural and emotional responses. 240 
Table 1

Mean standard deviation performance on executive tasks for high and low schizotypal groups

\begin{tabular}{|c|c|c|c|c|}
\hline \multirow[t]{2}{*}{ Test } & \multirow{2}{*}{$\frac{\text { High SPQ-B }}{n=(29)}$} & \multirow{2}{*}{$\frac{\text { Low SPQ-B }}{n=(32)}$} & \multirow[t]{2}{*}{$F$ value } & \multirow{2}{*}{$\frac{\text { Effect size }}{d(95 \% \mathrm{CI})}$} \\
\hline & & & & \\
\hline Letter fluency & $12.60(4.2)$ & $12.02(3.5)$ & $F=1.84, \mathrm{~ns}$ & $-0.15(-.29$ to -.02$)$ \\
\hline Category fluency & $17.50(4.3)$ & $16.98(3.4)$ & $F=0.38, \mathrm{~ns}$ & $-0.13(-.31$ to -.04$)$ \\
\hline Zoo planning (s) & $98.48(78.2)$ & $79.19(51.3)$ & $F=0.93, \mathrm{~ns}$ & $-0.28(-.42$ to -.16$)$ \\
\hline Zoo drawing (s) & $122.31(53.2)$ & $119.56(58.9)$ & $F=0.11, \mathrm{~ns}$ & $-0.04(-.18$ to .08$)$ \\
\hline Hayling auto (s) & $4.31(5.9)$ & $6.66(7.7)$ & $F=1.64, \mathrm{~ns}$ & $0.34(.20$ to .47$)$ \\
\hline Hayling inhib (s) & $20.9(20.6)$ & $26.09(22.4)$ & $F=0.13, \mathrm{~ns}$ & $0.25(.36$ to -.14$)$ \\
\hline Total DEX score & $31.76(10.8)$ & $21.28(8.2)$ & $F=13.81, P<.001$ & $-1.07(-1.24$ to -.94$)$ \\
\hline DEX inhibition & $10.38(4.2)$ & $6.72(3.1)$ & $F=15.23, P<.001$ & $-1.01(-1.69$ to -0.46$)$ \\
\hline DEX intention & $7.21(3.3)$ & $5.37(2.3)$ & $F=6.35, P=.015$ & $-0.66(-1.22$ to -0.13$)$ \\
\hline DEX social regulation & $8.34(3.7)$ & $5.66(3.0)$ & $F=9.80, P=.002$ & $-0.82(-1.39$ to -0.28$)$ \\
\hline DEX abstract problem solving & $5.97(2.4)$ & $3.56(1.8)$ & $F=19.57, P=.001$ & $-1.15(-1.78$ to -0.58$)$ \\
\hline
\end{tabular}

The intentionality factor includes items concerning planning and decision-making problems. The social regulation factor consists of items relating to emotional and social behaviour, lack of insight. The abstract problem-solving factor consists of items such as abstract thinking problems, perseveration, confabulation, and variable motivation.

\section{Results}

The participants were divided into two non-overlapping groups based on the mean SPQ-B scores $(M=$ 26.31 ; S.D. $=10.78$ ) and the data for four participants at the midpoint were removed. This resulted in 32 participants in the low SPQ-B group $(M=3.8 ;$ S.D. $=1.7$; range $1-7)$ and 29 in the high SPQ-B group $(M=11.3$; S.D. $=2.7$; range $8-17$ ). The high and low schizotypal groups did not differ in years of education (14.89 vs. 15.1: $F<1$ ) or sex ratio (high: 24 female and 5 male; low: 27 female and 5 male). The low SPQ group was significantly older than the high SPQ group (19.97 vs. 23.31: $\left.F_{(1,59)}=6.26, P=.015\right)$ and so was included as a covariate in the analyses (although it made no difference to the outcomes). We recorded errors on the Zoo map and Hayling tasks, but the error rates were too low to analyse.
A one-way between groups MANOVA (involving all 264 six cognitive measures and age as covariate) revealed no 265 significant main effect for group on the omnibus test: 266 $F_{(6,53)}<1$. By contrast, a univariate ANCOVA for the 267 self-report DEX measure revealed a main group effect 268 with the high SPQ group reporting more executive 269 problems $\left(F_{(1,59)}=13.8, P<0.001\right)$. Effect sizes were 270 small, except for the DEX, which produced a large effect 271 size (see Table 1). Further analysis and comparison of 272 the high and low SPQ groups on the four subscales of 273 the DEX revealed significant group differences for each 274 factor.

275

\subsubsection{Schizotypy factors and executive performance 276}

To further investigate the relationship between SPQ 277 and executive functioning, we correlated the subscale 278 scores within the SPQ (disorganised, interpersonal, and 279 cognitive-perceptual) with the subject scores on each 280 executive task using Pearson's $r$.

281

The most notable finding was the large significant 282 correlation of DEX scores with both the cognitive- 283 perceptual syndrome and the disorganised syndrome 284 (the correlation with the interpersonal syndrome failed 285 to reach significance). The DEX also failed to correlate 286

Table 2

Correlations Pearson $(r)$ between executive test performance, SPQ-B subscales and total DEX score

\begin{tabular}{llccc}
\hline & SPQ cognitive-perceptual & SPQ interpersonal & SPQ disorganisation & Total DEX score \\
\hline Letter fluency & -.01 & -.18 & $.28^{*}$ & .16 \\
Category fluency & .17 & -.23 & .03 & .14 \\
Zoo (planning time) & .02 & .20 & -.01 & -.22 \\
Zoo (drawing time) & .14 & -.11 & -.05 & -.07 \\
Hayling (automatic time) & -.10 & -.11 & -.13 & -.05 \\
Hayling (inhibition time) & -.13 & .13 & .09 & -.10 \\
DEX score & $.40^{* *}$ & .24 & $.59^{* *}$ & -
\end{tabular}

$* P<0.05, * * P<0.01$. 
significantly with any of the executive measures (see Table 2).

\section{Discussion}

This study investigated whether undergraduate subjects scoring high on schizotypal traits show significantly worse performance on tests of executive functioning than low schizotypal scorers. High and low SPQ scorers failed to show differences on a variety of executive measures (letter and category fluency, the time taken to plan and draw a route, complete the Zoo map, the response time on the Hayling test for the connected and unconnected sentences). By contrast the high SPQ scorers did score significantly higher on the DEX i.e. a questionnaire tapping self-reported everyday executive problems; and this extended to all four factors of the questionnaire. Finally, the DEX scores were significantly correlated with scores on the disorganisation and cognitive-perceptual subscales of the SPQ. By contrast, the correlation between the DEX and the interpersonal subscale of the SPQ failed to reach significance.

It is unlikely that we failed to find any differences on the executive tasks because the 'high' schizotypal group was not extreme enough or because the study lacked power. Neither of these explanations would readily account for the very large effect size found in the same subjects for the DEX (post hoc power $=.99$ ). Of course, it remains possible that examination of a more extreme SPQ group might reveal some cognitive problems that emerge following the self-reported behavioural symptoms; however, our high and low group scores are comparable with those in previous work e.g. Mata et al., 2005; Compton et al., 2007. Additionally, of course, a different executive battery might be more sensitive to executive problems; however, we did choose timed tests to make measures as sensitive as possible. A recent study by Dinn et al. (2002) divided their healthy subjects into those scoring high, medium and low on the negative i.e. interpersonal subscale; or on the positive i.e. cognitive-perceptual features of the SPQ-B and examined for executive test performance differences. As in the current study, Dinn et al. (2002) found no differences for either subscale on measures of verbal fluency, TMT-A and B or the Stroop. Nonetheless, in the context of our finding enhanced awareness of executive problems, Dinn and colleagues did find self-rated differences on the Frontal Lobe Personality Scale (Grace and Malloy, 1992), which is a questionnaire tapping self-reported frontal lobe behaviours.

The failure to find impairments in executive funcioning in the high SPQ group is inconsistent with some previous studies (Spaulding et al., 1989; Daneluzzo 337 et al., 1998; Lenzenweger et al., 1991; Lyons et al., 338 1991; Raine et al., 1992a; Poreh et al., 1995; Suhr, 1997; 339 Vogelmaier et al., 1997; Tallent and Gooding, 1999). 340 Nevertheless, as noted in the Introduction, others have 341 found evidence of no executive dysfunction in schizo- 342 typal subjects (Condray and Steinhauer, 1992; Trestman 343 et al., 1995; Lin et al., 2000; Raine et al., 1992b; Diforio 344 et al., 2000; Spitznagel and Suhr, 2002; Avons et al., 345 2003). One possible reason for the mixed findings may 346 reflect the large reliance by many of the studies on the 347 WCST and especially the perseverative measure. In the 348 current study, we used a wide range of alternative exe- 349 cutive tests (verbal fluency, semantic fluency, Zoo map 350 test, and Hayling test), none of which found a significant 351 difference. It is certainly not the case that the executive 352 dysfunction in schizophrenic patients is restricted only 353 to the perseverative measures from the WCST (for a 354 review, Laws, 1999). It is also notable that a variety of 355 measures of schizotypy have been used in such studies 356 (however, only the SPQ-B taps the dimensions linked to 357 all DSM diagnostic criteria).

The finding that high schizotypal participants scored 359 higher on the DEX is perhaps surprising, since patients 360 with acquired brain damage and degenerative disorders 361 affecting the frontal lobes and moreover, schizophrenics 362 show anosognosia i.e. underestimate their own execu- 363 tive problems (e.g. Wilson et al., 1996; Evans et al., 364 1997). Nonetheless, since constructs such as 'self-moni- 365 toring' and 'insight' are central to the broad concept of 366 executive functioning, it is perhaps not surprising that 367 underestimation occurs in patients with schizophrenia 368 because of their lack of insight. Our finding of increased 369 self-reporting of executive problems in the high SPQ 370 group, however, accords with the notion that such indi- 371 viduals experience everyday problems and maintain a 372 high degree of insight about those difficulties. This 373 finding does, however, parallel a recent report of in- 374 creased DEX scores in subjects reporting high levels of 375 dissociative experiences. Hence both high schizotypals 376 and high dissociative subjects may be hypervigilant to 377 their executive failures (Bruce et al., in press). It is also 378 possible that typical measures of executive functioning 379 fail to capture the very real executive problems that 380 high schizotypal and high dissociative subjects report. 381 In this context, it is notable that patients with frontal 382 lesions may perform within the normal range on tests of 383 executive functioning, despite reporting experiencing 384 difficulties in their daily lives (e.g. Shallice and Burgess, 385 1991). These findings suggest that executive functions 386 may not solely be manifested at a cognitive level, mea- 387 sured by objective neuropsychological tests, but on a 388 
cognitive-behavioural level in daily-life tasks (Chan, 2001).

Since the two self-report measures correlated with each other, but not with cognitive test performance, this could be viewed as a possible response bias. Although some common variance overlap exists between the SPQ-B and the DEX, this accounted for less than $25 \%$ of the variance, suggesting a moderate overlap. It was notable that the DEX correlated significantly only the cognitive-perceptual and disorganisation subscales, but not with the interpersonal subscale. This suggests that the overlap with selfrated executive problems occurs more for the positive than the negative symptoms of schizotypy.

It is possible that because both scales are self-report based, that they may not be accurate reflections of an individual's psychological status and with no impairment on task performance, we might question the reliability of their responses or their insight. However, this is not unusual. For example, in several studies from the Edinburgh High Risk Study, examining young people who are at enhanced genetic risk of schizophrenia (Johnstone et al., 2000), no performance decrements have been found for the Hayling, Stroop and semantic fluency (Byrne et al., 1999; Whalley et al., 2004, 2005). Nonetheless, using fMRI, Whalley et al. (2004, 2005), they have found that compared to normal controls, those at high risk showed abnormal frontal lobe interactions when performing the Hayling test (even though no difference in test performance was noted). The latter suggests that abnormal brain activity may precede the appearance measurable cognitive disturbance. This might be viewed as consistent with the current finding of a dissociation between intact executive test performance and high selfrating of executive behaviours in everyday life. In other words, a heightened self-awareness of executive behavioural problems and even functional brain changes in high schizotypal subjects may precede the onset of clear deficits on executive tests themselves.

In summary, we have found no evidence of poor performance on executive tests in participants scoring highly on the SPQ-B. By contrast, the high SPQ group and in particular high scorers on the cognitive-perceptual and disorganisation features of schizotypy scored significantly higher on the DEX questionnaire. Heightened awareness of everyday executive dysfunction in schizotypal subjects was therefore more associated with the positive than the negative features of schizotypy. The finding of good levels of insight (into executive problems) in high schizotypal subjects suggests that the poor insight typically associated with schizophrenia may emerge only after illness onset (see Simon et al., 2006).

\section{Uncited references}

Bennett et al., 2005

Bogod et al., 2003

Chen et al., 2000

Krabbendam et al., 1999

Raine, 2005

\section{References}

Aleman, A., Agrawal, N., Morgan, K.D., David, A.S., 2006. Insight in 448 psychosis and neuropsychological function: meta-analysis. British 449 Journal of Psychiatry 189, 204-212.

Avons, S.E., Nunn, J.A., Chan, L., Armstrong, H., 2003. Executive 451 functioning assessed by memory updating and random generation 452 in schizotypal individuals. Psychiatry Research 120, 145-154. 453

Axelrod, S.R., Grilo, C.M., Sanislow, C., McGlashan, T.H., 2001. 454 Schizotypal personality questionnaire-brief: factor structure and 455 convergent validity in inpatient adolescents. Journal of Personality 456 Disorders $15,168-179$.

Bennett, P.C., Ong, B., Ponsford, J., 2005. Measuring executive-dys- 458 function in an acute rehabilitation-setting: using the dysexecutive 458 questionnaire DEX. Jeurnal of the International Neurepsycheleg- 460 ical Society $11,376 \quad 385$.

Benton, A.L., Hamsher, K. deS., 1976. Multilingual Aphasia Exami- 462 nation. University of Iowa, Iowa City.

Boged, N.M., Mateer, C.A., Maedonald, S.W.S., 2003. Self-awareness 464 after traumatic brain injury: a comparison of measures and their 465 relationship to executive function. Journal of International Neuro- 466 psychologieal Society 9, $450-458$.

Bruce, A.S., Ray, W.J., Bruce, J.M., Arnett, P.A., Carlson, R.A., in press. 468 The relationship between executive functioning and dissociation. 469 Journal of Clinical and Experimental Neuropsychology. 470

Burgess, P.W., Shallice, T., 1997. The Hayling and Brixton Tests. 471 Thames Valley Test Company, Bury St. Edmunds. 472

Byrne, M., Hodges, A., Grant, E., Owens, D.C., Johnstone, E.C., 1999. 473 Neuropsychological assessment of young people at high genetic 474 risk for developing schizophrenia compared with controls: prelimi- 475 nary findings of the Edinburgh High Risk Study EHRS. Psycho- 476 logical Medicine 29, 1161-1173.

Chan, R.C., 2001. Dysexecutive symptoms among a non-clinical 478 sample: a study with the use of Dysexecutive Questionnaire. Bri- 479 tish Journal of Psychology 92, 551-565.

Chen, Y.L.R., Erie, Y.H., Felice, M., Lieh, 2000. Semantic verbat 481 flueney deficit as a familial trait marker in schizophrenia. Psychiat- 482 ry Research 95,133148 .

Cohen, J., 1988. Statistical Power for the Behavioural Sciences. 2nd edn. 484 Lawrence Erlbaum Associates, Hillsdale, NJ.

Condray, R., Steinhauer, S.R., 1992. Schizotypal personality disorder 486 in individuals with and without schizophrenic relatives: similarities 487 and contrasts. Schizophrenia Research 7, 33-41.

Compton, M.T., Chien, V.H., Bollini, A.M., 2007. Psychometric 489 properties of the Brief Version of the Schizotypal Personality 490 Questionnaire in relatives of patients with schizophrenia-spectrum 491 disorders and non-psychiatric controls. Schizophrenia Research 492 91, 122-131.

Daneluzzo, E., Bustini, M., Stratta, P., Casacchi, M., Rossi, A., 1998. 494 Schizotypal personality questionnaire and Wisconsin Card Sorting 495 Test in a population of DSM-III-R schizophrenic patients and 496 controls. Comprehensive Psychiatry 39, 143-148. 
Dinn, W.M., Harris, C.L., Aycicegi, A., Green, P., Andover, M.S., 2002. Positive and negative schizotypy in a student sample: neurocognitive and clinical correlates. Schizophrenia Research 56, 171-185.

Evans, J.J., Chua, E., McKenna, P.J., Wilson, B.A., 1997. Assessment of the dysexecutive syndrome in schizophrenia. Psychological Medicine 27, 635-646.

Goodglass, H., Kaplan, E., 1972. Assessment of Aphasia and Related Disorders. Lea and Febiger, Philadelphia.

Gooding, D.C., Kwapil, T.R., Tallent, K.A., 1999. Wisconsin Card Sorting Test in schizotypic individuals. Schizophrenia Research 40, 201-209.

Heinrichs, R.W., Zakzanis, K.K., 1998. Neurocognitive deficit in schizophrenia: a quantitative review of the evidence. Neuropsychology 12, 426-445.

Hill, K., Mann, L., Laws, K.R., Stephenson, C.M., Nimmo-Smith, I., McKenna, P.J., 2004. Hypofrontality in schizophrenia: a metaanalysis of functional imaging studies. Acta Psychiatrica Scandinavica $110,243-256$

Jahshan, C.S., Sergi, M.J., 2007. Theory of mind, neurocognition and functional status in schizotypy. Schizophrenia Research 89, 278-286.

Johnstone, E.C., Abukmeil, S.S., Byrne, M., Clafferty, R., Grant, E., Hodges, A., Lawrie, S.M., Owens, D.G.C., 2000. Edinburgh high risk study - findings after four years: demographic, attainment and psychopathological issues. Schizophrenia Research 46, 1-15.

Kiang, M., Kutos, M., 2006. Abnormal typicality of responses on a category fluency task in schizotypy. Psychiatry Research 145, $119-126$.

Krabbendam, L., de Vugt, M.E., Derix, M.M.A., Jolles, J., 1999. The Behavioural Assessment of the Dysexecutive Syndrome as a tool to assess executive functions in schizophrenia. Clinical Neuropsy ehologist $13,370-375$.

Laws, K.R., 1999. A meta-analytic review of Wisconsin Card Sort Studies in schizophrenia: general intellectual deficit in disguise? Cognitive Neuropsychiatry 4, 1-35.

Lenzenweger, M., Korfine, L., 1994. Perceptual aberrations, schizotypy, and the Wisconsin Card Sorting Test. Schizophrenia Bulletin 20, 345-357.

Lenzenweger, M.F., Cornblatt, B.A., Putnick, M., 1991. Schizotypy and sustained attention. Journal of Abnormal Psychology 100, 84-89.

Lin, C.C.H., Chen, W.J., Yang, H-J., Hsiao, C.K., Tien, A.Y., 2000. Performance on the Wisconsin Card Sorting Test among adolescents in Taiwan: norms, factorial structure and relation to schizotypy. Journal of Clinical and Experimental Neuropsychology $22,69-79$.

Lyons, M.J., Meria, M.E., Young, L., Kremen, W.S., 1991. Impaired cognitive functioning in symptomatic volunteers with schizotypy: preliminary findings. Biological Psychiatry 30, 424-426.

Mata, I., Mataix-Cols, D., Peralta, 2005. Schizotypal Personality Questionnaire-Brief: factor structure and influence of sex and age in a nonclinical population. Personality and Individual Differences 38, 1183-1192.

Mitropoulou, V., Harvey, P.D., Maldari, L.A., Moriarty, P.J., New, A.S., Silverman, J.M., Siever, L.J., 2002. Neuropsychological performance in schizotypal personality disorder: evidence regarding diagnostic specificity. Biological Psychiatry 52, 1175-1182.

Mooney, B., Walmsley, C., McFarland, K., 2006. Factor analysis of the self-report dysexecutive (DEX-S) questionnaire. Applied Neuropsychology $13,12-18$.

Poreh, A.M., Ross, T.P., Whitman, R.D., 1995. Reexamination of executive functions in psychosis-prone college students. Personality and Individual Differences 18, 535-539.
Raine, A., 1991. The SPQ: a scale for the assessment of schizotypal 560 personality based on DSM-III-R criteria. Schizophrenia Bulletin 561 $17,556-564$.

Raine, A., 2005大 Schizotypal personality: neurodevelopmental and 563 psychosocial trajectories. Annual Review in Clinical Psychology 564 2, 291-326.

Raine, A., 2006. Schizotypal personality: neurodevelopmental and 566 psychosocial trajectories. Annual Review of Clinical Psychology 567 2, 291-326.

568

Raine, A., Benishay, D., 1995. The SPQ-B: a brief screening instru- 569 ment for schizotypal personality disorder. Journal of Personality 570 Disorders 9, 346-355.

571

Raine, A., Triphon, N., Kim, D., Hesler, A., Bird, L., Lencz, T., 572 Redmon, M., Scerbo, A., 1992a. Schizotypal personality: factor 573 structure, sex differences, psychiatric differences, genetics, psy- 574 chophysiology and neuropsychology. Paper presented at the 1992575 Western Psychological Association Meeting, Portland, Ore. $\quad 576$

Raine, A., Sheard, C., Reynolds, G.P., Lencz, T., 1992b. Pre-frontal 577 structural and functional deficits associated with individual dif- 578 ferences in schizotypal personality. Schizophrenia Research 27, 579 45-53. 580

Shallice, T., Burgess, P.W., 1991. Deficits in strategy applications 581 following frontal lobe damage in man. Brain 114, 727-741. 582

Simon, A.E., Begrer, G.E., Giacomini, V., Ferrero, F., Mohr, S., 2006. 583 Insight, symptoms and executive function in schizophrenia. Cog- 584 nitive Neuropsychiatry 11, 437-451. 585

Sitskoorn, M.M., Aleman, A., Ebisch, S.J.H., Apples, M.C.M., Kahn, 586 R.S., 2004. Cognitive deficits in relatives of patients with schizo- 587 phrenia: a meta-analysis. Schizophrenia Research 71, 285-295. 588

Szöke, A., Schürhoff, F., Mathieu, F., Meary, A., Ionescu, S., Leboyer, 589 M., 2005. Tests of executive function in first-degree relatives of 590 schizophrenic patients: a meta-analysis. Psychological Medicine 591 $35,771-782$.

Spaulding, W., Garbin, C.P., Dras, S.R., 1989. Cognitive abnormalities 593 in schizophrenic patients and schizotypal college students. Journal 594 of Nervous Mental Disease 177, 717-728.

Spitznagel, M.B., Suhr, J.A., 2002. Executive function deficits 596 associated with symptoms of schizotypy and obsessive-compul- 597 sive disorder. Psychiatry Research 110, 151-163. 598

Suhr, J.A., 1997. Executive functioning deficits in hypothetically 599 psychosis-prone college students. Schizophrenia Research 27, 600 29-35.

Tallent, K.A., Gooding, D.C., 1999. Working memory and Wisconsin 602 Card Sorting Test performance in schizotypic individuals: a 603 replication and extension. Psychiatry Research 89, 161-170. 604

Vogelmaier, M.M., Siedman, L., Salisbury, D., McCarly, R.W., 1997. 605 Neuropsychological dysfunction in schizotypal personality disor- 606 der: a profile analysis. Biological Psychiatry 41, 530-540. 607

Whalley, H.C., Simonotto, E., Flett, S., Marshall, I., Ebmeier, K.P., 608 Owens, D.G.C., Goddard, N.H., Johnstone, E.C., Lawrie, S.M., 609 2004. fMRI correlates of state and trait effects in subjects at 610 genetically enhanced risk of schizophrenia. Brain 127, 478-490. 611

Whalley, H.C., Simonotto, E., Flett, S., Marshall, I., Owens, D.G.C., 612 Goddard, N.H., Johnstone, E.C., Lawrie, S.M., 2005. Functional 613 disconnectivity in subjects at high genetic risk of schizophrenia. 614 Brain 128, 2097-2108.

Wilson, B.A, Alderman, N., Burgess, P.W, Emslie, H., Evans, J.J., 616 1996. Behavioural Assessment of the Dysexecutive Syndrome. 617 Thames Valley Test Company, Bury St Edmunds, England. 